\title{
The CEDAR Study: A Longitudinal Study of the Clinical Effects of Conventional DMARDs and Biologic DMARDs in Australian Rheumatology Practice
}

\author{
Lynden Roberts, ${ }^{1}$ Kathleen Tymms, ${ }^{2}$ Julien de Jager, ${ }^{3}$ \\ Geoffrey Littlejohn, ${ }^{1}$ Hedley Griffiths, ${ }^{4}$ Dave Nicholls, ${ }^{5}$ Paul Bird, ${ }^{6}$ \\ Jennifer Young, ${ }^{7}$ Julie Hill, ${ }^{8}$ and Jane Zochling ${ }^{9}$ \\ ${ }^{1}$ Department of Medicine, Monash University, Clayton, VIC, Australia \\ ${ }^{2}$ Rheumatology Department, Australian National University, Canberra, ACT, Australia \\ ${ }^{3}$ Pacific Private Clinic, Southport, QLD, Australia \\ ${ }^{4}$ Barwon Rheumatology Service, Geelong, VIC, Australia \\ ${ }^{5}$ Coast Joint Care and University of the Sunshine Coast, Sunshine Coast, QLD, Australia \\ ${ }^{6}$ University of New South Wales, Sydney, NSW, Australia \\ ${ }^{7}$ Roche Products Pty Limited, Sydney, NSW, Australia \\ ${ }^{8}$ McCloud Consulting Group, Sydney, NSW, Australia \\ ${ }^{9}$ Menzies Institute for Medical Research, Hobart, TAS, Australia \\ Correspondence should be addressed to Lynden Roberts; lynden.roberts@monash.edu
}

Received 3 February 2017; Revised 13 April 2017; Accepted 27 April 2017; Published 23 May 2017

Academic Editor: Ruben Burgos-Vargas

Copyright (c) 2017 Lynden Roberts et al. This is an open access article distributed under the Creative Commons Attribution License, which permits unrestricted use, distribution, and reproduction in any medium, provided the original work is properly cited.

Objectives. To observe the choices of conventional disease modifying antirheumatic drugs (cDMARDs) and biologic DMARDs (bDMARDs) in the management of rheumatoid arthritis (RA) in Australian routine clinical practice, to assess treatment survival and determine the effect of cDMARDs/bDMARDs on disease activity. Methods. Routinely collected, deidentified clinical data was sourced from 20 Australian rheumatology practices. RA patients aged $\geq 18$ years, who had received cDMARDs/bDMARDs and a recorded subsequent visit, were included. A linear mixed model was used to determine the change over time and the percentage reduction in disease activity was summarized. Results. 12,526 RA patients were included: $72 \%$ females, mean age 62 years. cDMARDs and bDMARDs were used in $92 \%$ and $30 \%$ of patients, respectively. The most commonly prescribed cDMARD was methotrexate (76\% patients); median time to stopping treatment was 337 months [95\% CI: 279-ND]. Etanercept was the most commonly prescribed bDMARD (12\% patients); median time to stopping treatment was 79 months [95\% CI: 57-93]. Of 5,341 patients with a first change in medication (cDMARD or bDMARD), $87 \%$ had therapy escalation and $13 \%$ deescalation. Reduction in DAS28-ESR, 6-month post-DMARDs initiation ranged from 3\%, adalimumab, to 14\%, leflunomide and tocilizumab. Conclusions. In this large Australian cohort of unselected community RA patients, the choices of cDMARDs/bDMARDs are aligned with current international guidelines.

\section{Introduction}

High-quality clinical research underpins our knowledge of the efficacy of various rheumatoid arthritis (RA) therapies [1-4]. Randomized clinical trials (RCTs) have provided reassuring safety and efficacy evidence to support the use of the numerous new therapeutic options for RA over the last 20 years [1-3]. However, in real world practice, outside of the controlled environment of these RCTs, additional factors are likely to influence the use of conventional disease modifying antirheumatic drugs (cDMARDs) and biologic DMARDs (bDMARDs).

RCTs are conducted on carefully selected groups of patients under strict clinical conditions; therefore, it is 
unclear how the efficacy and safety signals observed in the RCTs will translate into the uncontrolled, relatively unselected environment of routine clinical practice.

There are limited routine clinical practice data available that assess the uptake of new treatments in RA patients $[5,6]$. Such data would be useful to better understand any barriers to the implementation of new evidence and would be likely to inform strategies designed to enhance rational prescribing.

The OPAL-QUMI (Optimising Patient outcomes in Australian rheumatoLogy-Quality Use of Medicines Initiative) consortium [7] is a point of care-derived observational registry database with clinical data recorded by Australian rheumatologists in real-time during routine clinical encounters. Cross-sectional data from OPAL has helped to provide insights into the reasons why a proportion of RA patients remain in moderate disease activity using a treat-to-target strategy [8].

\section{Methods}

2.1. Study Design. CEDAR is a multicentre, longitudinal, observational study. The primary objective of the study was to identify the choices of cDMARDs/bDMARDs being made over time for the management of RA in Australian patients. The secondary objectives were to assess the survival of RA patients on treatment and the effects of cDMARDs/bDMARDs on disease activity.

The study which was approved by the University of New South Wales Human Research Ethics Committee (reference number HC12094) was conducted in accordance with local guidelines, as set out in the National Statement on Ethical Conduct in Human Research.

2.2. Inclusion Criteria. Patients diagnosed with RA, aged $\geq 18$ years, who had received any medication, including cDMARDs, bDMARDs, corticosteroids, or NSAIDs, either as monotherapy or in addition to their stable previous therapy, and who also had subsequent follow-up visits as well as a change in cDMARDs/bDMARDs recorded were eligible to be included in the study. Patients who requested their data not to be collected for research purposes were excluded from the analyses.

2.3. Data Capture. The study was conducted by the OPALQUMI using the clinical software program Audit4 (described previously) [7]. Deidentified data was sourced from 20 participating rheumatology practices comprising 42 rheumatologists across Australia. Data was captured in May 2012. For the assessment of treatment effect on disease activity, the baseline data was collected in May 2012 and 6-month data was collected in November 2012.

2.4. Clinical Observations. Parameters collected included demographics (gender and age), diagnosis (clinical onset of RA and disease duration), disease measures (including DAS28-ESR), tender joint count (TJC), swollen joint count (SJC), and laboratory observations (inflammatory markers, autoantibody status, RF and/or ACPA positivity) and medications (NSAIDs, steroids, cDMARDs and bDMARDs with initiation dates, duration of treatment, and reason for cessation).

However, being point of care clinical data, there are parameters missing for some analyses (e.g., effect of treatment on disease activity) in these cases; we have highlighted/defined the number of patients included.

\subsection{Analytical Assessments and Statistical Considerations,} Medication change was analyzed in 9 prespecified categories: methotrexate monotherapy, combination of $>1$ cDMARDs, combination of methotrexate and leflunomide, monotherapy of cDMARDs other than methotrexate, monotherapy of bDMARDs, combination of bDMARDs plus methotrexate, combination of bDMARDs plus cDMARDs other than methotrexate, combination of bDMARDs and $>1$ cDMARDs, and no cDMARDs/bDMARDs therapy. Treatment escalation was defined as a new cDMARDs/bDMARDs being introduced and treatment deescalation was defined as the cessation of cDMARDs/bDMARDs.

Treatment survival was defined as the number of months from the first prescription of the drug until the last known date taking the drug. Survival on treatment was analyzed for the most frequently recorded cDMARDs (methotrexate, hydroxychloroquine, leflunomide, and sulfasalazine) and bDMARDs (etanercept, adalimumab, tocilizumab, and abatacept). The number of patients on other biologics was too small for any meaningful analyses to be carried out. Government subsidized bDMARDs sequentially became available in Australia for etanercept in 2003, adalimumab in 2004, abatacept in 2008, and tocilizumab in 2010. Hence, data for tocilizumab, in particular, was over a shorter timeframe in this study. The EULAR response criteria were defined according to van Gestel et al., 1996 [9].

Descriptive statistics [mean, standard deviation (SD), and range] were provided for continuous variables and frequency counts for categorical variables. The median and distribution of treatment survival were estimated using Kaplan-Meier methodology. A linear mixed model was used to determine change over time and all medications with adequate sample size were included. The assumptions of the linear mixed model were that the observations are normally distributed with a constant variance and independence. The assumptions were assessed graphically.

\section{Results}

3.1. Patients. A total of 12,526 RA patients were screened: $72 \%$ females, mean age 62 years (range 18-100 years). Demographics and disease characteristics for the bDMARDs and bDMARDs groups are presented in Table 1 . There were $57 \%$ of patients with a baseline DAS28-ESR measurement and the mean score was 3.2 (SD 1.56). RF and ACPA positivity rate was not available for the study; diagnosis of rheumatoid arthritis was made by the treating rheumatologist.

3.2. Prescribed Medications. Ninety-two percent $(n=11,511)$ of patients had at least one cDMARD, 30\% $(n=3,697)$ had at least one bDMARD, 58\% $(n=7,223)$ had an oral corticosteroid, and $30 \%(n=3,699)$ had at least one NSAID (mainly 

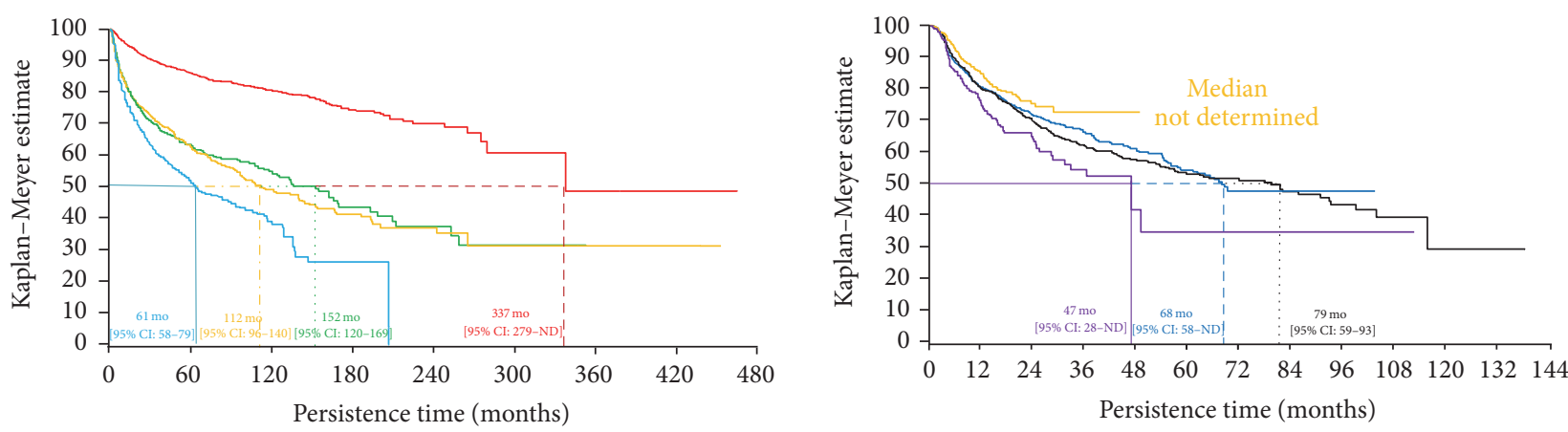

$\begin{array}{lcccccccc}\begin{array}{l}\text { Patients remaining } \\ \text { Methotrexate }\end{array} & 9508 & 1345 & 565 & 192 & 70 & 11 & 1 & 0 \\ \text { Hydroxychloroquine } & 3527 & 245 & 96 & 36 & 15 & 3 & 1 & 0 \\ \text { Leflunomide } & 3275 & 261 & 53 & 1 & 0 & 0 & 0 & 0 \\ \text { Sulfasalazine } & 2340 & 326 & 131 & 59 & 21 & 5 & 1 & 0 \\ & \\ & \\ & & \\ & & & \\ & \end{array}$

(a)

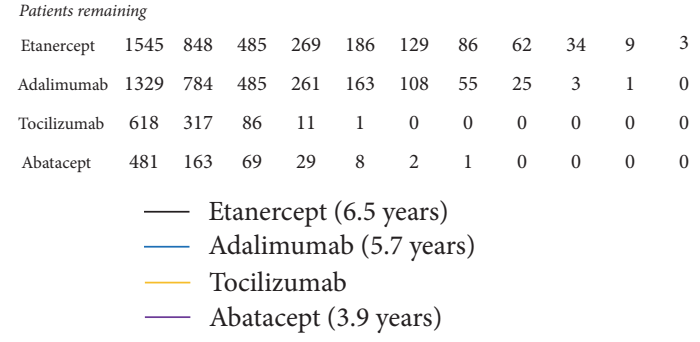

(b)

FIGURE 1: Kaplan-Meier plot of treatment survival: (a) cDMARDs and (b) bDMARDs. ND = not determined.

TABle 1: Patient demographics and disease characteristics.

\begin{tabular}{ccccc}
\hline & $n$ & Mean & SD & Min-max \\
\hline Age (years) & 12,526 & 61.9 & 14.0 & $18.0-100.0$ \\
cDMARDs $^{\#}$ & 11,511 & 61.8 & 13.9 & $18.0-99.0$ \\
bDMARDs $^{*}$ & 3,697 & 59.0 & 13.1 & $19.0-91.0$ \\
Disease duration (years) & 9,069 & 11.1 & 10.6 & $0-77.4$ \\
cDMARDs & 8,591 & 11.1 & 10.6 & $0-77.4$ \\
bDMARDs & 2,387 & 13.7 & 10.5 & $0.3-61.1$ \\
DAS28-ESR & 7,112 & 3.2 & 1.6 & $0-8.8$ \\
cDMARDs & 6,728 & 3.2 & 1.6 & $0-8.8$ \\
bDMARDs & 2,576 & 3.4 & 1.7 & $0-8.7$ \\
Tender joint count & 8,842 & 3.6 & 5.6 & $0-28.0$ \\
cDMARDs & 8,285 & 3.6 & 5.5 & $0-28.0$ \\
bDMARDs & 3,057 & 4.4 & 6.3 & $0-28.0$ \\
Swollen joint count & 8,842 & 3.6 & 5.5 & $0-28.0$ \\
cDMARDs & 8,285 & 3.7 & 5.5 & $0-28.0$ \\
bDMARDs & 3,057 & 4.2 & 6.1 & $0-28.0$ \\
Rheumatoid factor & 4,115 & 137.4 & 297.5 & $0-5088.0$ \\
cDMARDs & 3,846 & 139.4 & 300.0 & $0-5088.0$ \\
bDMARDs & 1221 & 158.9 & 344.1 & $0-4886.0$ \\
ACPA & 2282 & 110.5 & 198.3 & $0-2000.0$ \\
cDMARDs & 2137 & 110.5 & 193.0 & $0-2000.0$ \\
bDMARDs & 726 & 124.7 & 210.4 & $0-1816.0$ \\
\hline
\end{tabular}

$\mathrm{SD}=$ standard deviation; ${ }^{*}$ patients taking at least one cDMARD; ${ }^{*}$ patients taking at least one bDMARD.

meloxicam and celecoxib). The most commonly prescribed cDMARDs and bDMARDs were methotrexate $(75.9 \% ; n=$ $9,508)$ and etanercept $(12.3 \% ; n=1,545)$, respectively, with all prescribed cDMARDs and bDMARDs presented in Table 2.
3.3. Treatment Survival. The treatment survival curves are presented in Figures 1(a) and 1(b). The median time to stopping treatment was 337 months [95\% CI: $279-\mathrm{ND}$ ] for methotrexate, 152 months [95\% CI: 120-168] for hydroxychloroquine, 112 months [95\% CI: 96-140] for sulfasalazine, and 61 months [95\% CI: 58-79] for leflunomide, while, for patients receiving bDMARDs, the median time to stopping treatment was 79 months [95\% CI: 57-93] for etanercept, 68 months [95\% CI: 58-ND] for adalimumab, and 47 months [95\% CI: $28-\mathrm{ND}$ ] for abatacept. The median time to stopping tocilizumab was not reached at the time of data analysis. For patients commencing cDMARDs, Kaplan-Meier (KM) estimates show that 95\% [95\% CI: 94.5-95.5] of the patients who commenced on methotrexate, 82\% [95\% CI: 80.7-83.6] on hydroxychloroquine, 77\% [95\% CI: 75.0-78.2] on leflunomide, and $82 \%$ [95\% CI: 80.1-83.7] on sulfasalazine persisted on the treatment at 12 months.

Similarly for bDMARDs, KM estimates show that $80 \%$ [95\% CI: 77.9-82.4] of patients who commenced on etanercept, $81 \%$ [95\% CI: 78.2-82.9] on adalimumab, 85\% [95\% CI: 81.5-88.0] on tocilizumab, and 75\% [95\% CI: 70.0-80.1] on abatacept persisted on the treatment at 12 months.

3.4. Medication Choice over Time. A total of 5,341 patients had a first change in medication after receiving their initial RA medication. Of these, 4,660 (87\%) had their treatment escalated and 681 (13\%) had their treatment deescalated. The most common first therapeutic RA medication changes were treatment escalations from monotherapy methotrexate, monotherapy cDMARDs other than methotrexate, and monotherapy bDMARDs (Figure 2(a)).

Two thousand one hundred and eighty-four patients had a second change in their medication; 1,244 (57\%) 

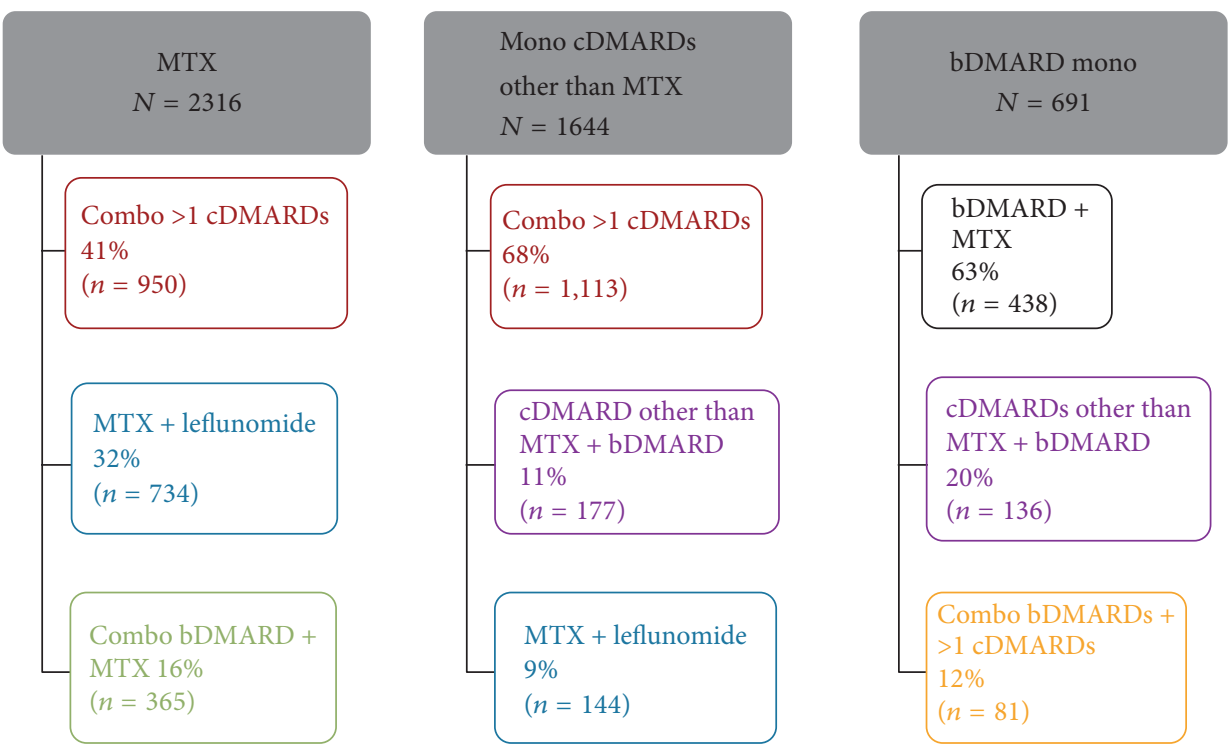

(a)
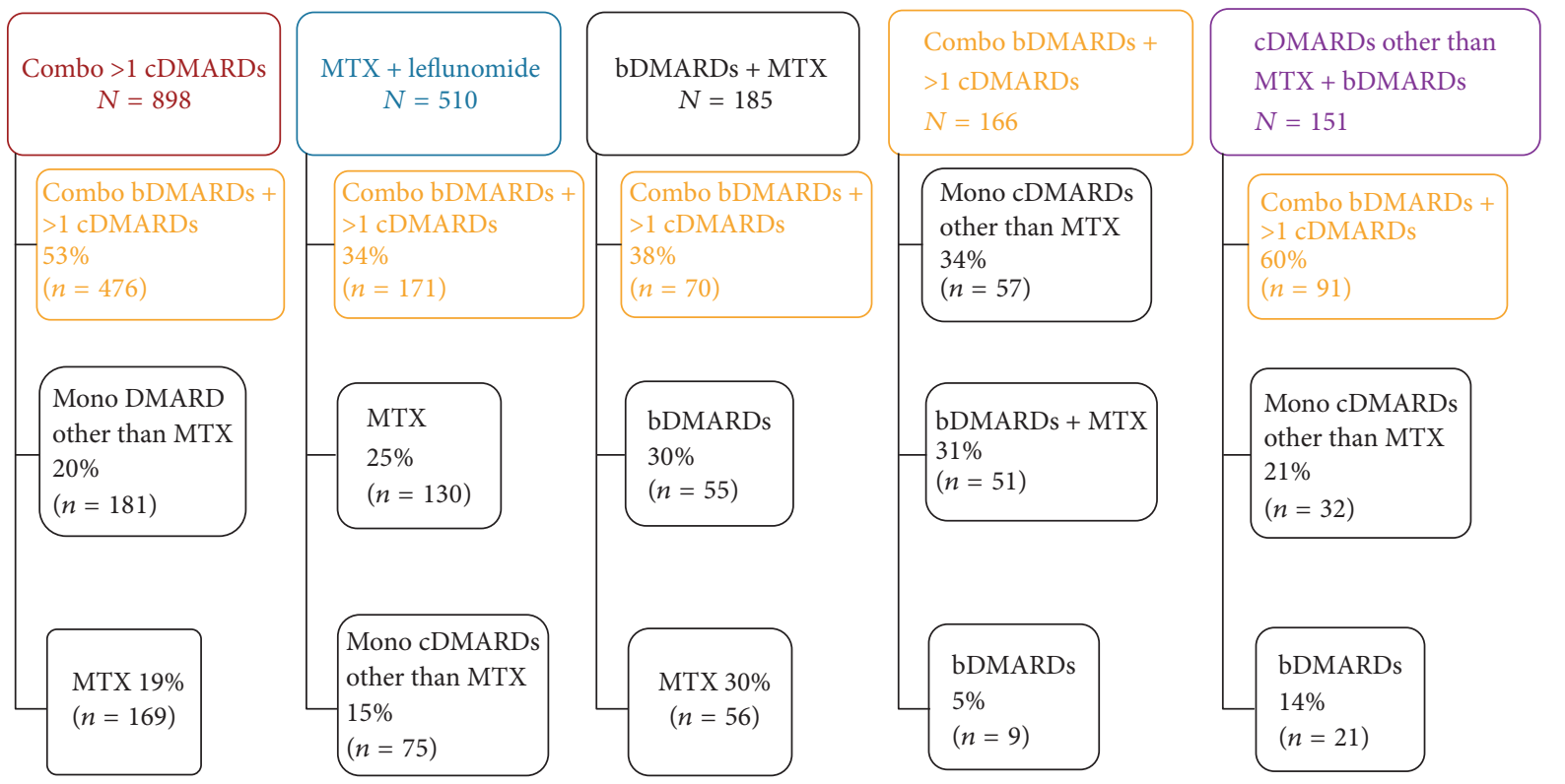

(b)

FIGURE 2: Most common treatment changes in RA medications at first and second change. (a) Treatment algorithm observed when DMARDs were first changed. (b) Treatment algorithm observed when DMARDs were changed a second time. Boxed groups at the top of each figure represent the patient's medication group prior to the medication change. The lines lead to boxes beneath that represent the distribution of medication groups after the medication change. Combo $=$ combination; mono $=$ monotherapy; MTX $=$ methotrexate. Percentages may not add up to $100 \%$ as only the most common changes are included.

had their treatment escalated and 940 (43\%) had their treatment deescalated. The most common treatment changes in RA medications at a second change are shown in Figure 2(b), which illustrates five of the most common decisions (combination of more than one cDMARD, methotrexate plus leflunomide, bDMARDs plus methotrexate, combination of bDMARDs plus more than one CDMARD, and CDMARDs other than methotrexate plus bDMARDs).
3.5. Disease Activity. Over a 6-month period, the change in disease activity was assessed following the commencement of a DMARD. Statistically significant, although clinically modest, reductions in mean DAS28-ESR were observed for methotrexate (9.4\%), hydroxychloroquine (9.6\%), leflunomide $(13.8 \%)$, sulfasalazine $(12.9 \%)$, etanercept $(8.9 \%)$, and tocilizumab (13.9\%). A reduction in DAS28-ESR (3.4\%) was also observed with adalimumab; however, this was not statistically significant (Table 3). 
TABLE 2: Distribution of treatments in the total patient cohort $(N=12,526)$. Multiple occurrences of the same medication in one individual were counted once.

\begin{tabular}{lccc}
\hline cDMARDs & $n(\%)$ & bDMARDs & $n(\%)$ \\
\hline Patients with at least one treatment & $11,511(91.9 \%)$ & Patients with at least one treatment & $3,697(29.5 \%)$ \\
\hline Methotrexate & $9,508(75.9 \%)$ & Etanercept & $1,545(12.3 \%)$ \\
Hydroxychloroquine & $3,527(28.2 \%)$ & Adalimumab & $1,329(10.6 \%)$ \\
Leflunomide & $3,275(26.1 \%)$ & Tocilizumab & $618(4.9 \%)$ \\
Sulfasalazine & $2,340(18.7 \%)$ & Abatacept & $481(3.8 \%)$ \\
Sodium aurothiomalate & $114(0.9 \%)$ & Golimumab & $376(3.0 \%)$ \\
Azathioprine & $98(0.8 \%)$ & Rituximab & $339(2.7 \%)$ \\
Cyclosporine & $89(0.7 \%)$ & Certolizumab pegol & $273(2.2 \%)$ \\
Auranofin & $43(0.3 \%)$ & Infliximab & $68(0.5 \%)$ \\
Penicillamine & $28(0.2 \%)$ & Anakinra & $3(<1.0 \%)$ \\
Cyclophosphamide & $10(0.1 \%)$ & & \\
\hline
\end{tabular}

TABLE 3: Change in DAS28-ESR scores over a 6-month period.

\begin{tabular}{|c|c|c|c|c|c|}
\hline $\begin{array}{l}\text { Treatment } \\
\text { as monotherapy or in combination }\end{array}$ & $n$ & $\begin{array}{c}\text { Month } 0 \\
\text { mean (SEM) }\end{array}$ & $\begin{array}{c}\text { Month } 6 \\
\text { mean (SEM) }\end{array}$ & $\begin{array}{l}\text { Change over time } \\
\text { value }(\%)\end{array}$ & $P$ value \\
\hline Methotrexate & 1,850 & $3.19(0.03)$ & $2.89(0.03)$ & $0.30(9.4 \%)$ & $<0.001$ \\
\hline Hydroxychloroquine & 494 & $3.33(0.06)$ & $3.01(0.06)$ & $0.32(9.6 \%)$ & $<0.001$ \\
\hline Leflunomide & 472 & $3.53(0.07)$ & $3.04(0.06)$ & $0.49(13.8 \%)$ & $<0.001$ \\
\hline Sulfasalazine & 319 & $3.55(0.09)$ & $3.09(0.08)$ & $0.46(12.9 \%)$ & $<0.001$ \\
\hline Adalimumab & 324 & $2.96(0.07)$ & $2.86(0.06)$ & $0.10(3.4 \%)$ & 0.106 \\
\hline Etanercept & 394 & $3.15(0.07)$ & $2.87(0.06)$ & $0.28(8.9 \%)$ & $<0.001$ \\
\hline Tocilizumab & 128 & $2.51(0.112)$ & $2.16(0.10)$ & $0.35(13.9 \%)$ & 0.001 \\
\hline
\end{tabular}

EULAR responses at 6 months were analyzed for 9 medications (as combination or monotherapy): methotrexate, hydroxychloroquine, leflunomide, sulfasalazine, etanercept, adalimumab, tocilizumab, abatacept, and rituximab, with the remaining medications not having sufficient data to record a EULAR response (Table 4).

\section{Discussion}

This study describes the use of cDMARDs/bDMARDs and medication survival in a large cohort of unselected community RA patients. Conventional DMARD usage reflected the contemporary treatment paradigm with methotrexate, hydroxychloroquine, leflunomide, and sulfasalazine being the predominant cDMARDs used. Over one-quarter of patients in this study used leflunomide, which is a higher rate of usage than what has been observed in Europe and North America [10, 11]. This may be because leflunomide is one of the CDMARD options listed for use prior to a patient accessing government funded biologics in Australia. For bDMARDs, almost $30 \%$ of Australian RA patients had been exposed to at least one bDMARD with etanercept, adalimumab, tocilizumab, and abatacept being the most common.

Treatment survival was long for all the studied cDMARDs. Methotrexate had the longest treatment survival and appeared to be fulfilling a role as an anchor medication, as it was also the most commonly used medication. The median time on treatment was shorter for bDMARDs compared to cDMARDs. This may be because, in Australia, patients must fail to respond to at least $2 \mathrm{cDMARDs}$ prior to accessibility to government funded bDMARDs. Accordingly, the patient group receiving bDMARDs in Australia are selected as nonresponders and therefore may represent a group with more refractory disease compared to the unselected group of patients receiving cDMARDs. It is to be expected that the response rates in this more refractory group might therefore be reduced. Nevertheless, the bDMARD median survival rates were similar to those seen in an Italian registry cohort [12].

Australian rheumatologists appear to closely follow the EULAR treatment guidelines for use of medications in the treatment of RA [4]. At the first medication change in patient treatment, the most common escalation pathway was from monotherapy methotrexate. The most common second medication change was the addition of a bDMARD to the combination cDMARDs regimens. These pathways are generally consistent with both the NICE and ACR guidelines updated in 2013 and 2012, respectively [13, 14], with the exception that, in OPAL patients, initial monotherapy cDMARD treatment is considerably more common than initial combination therapy.

The modest reductions in DAS28-ESR over 6 months for the bDMARDs in the OPAL patient cohort appear to be lower than what might have been expected based on previously reported RCTs [15-18] and other observational 


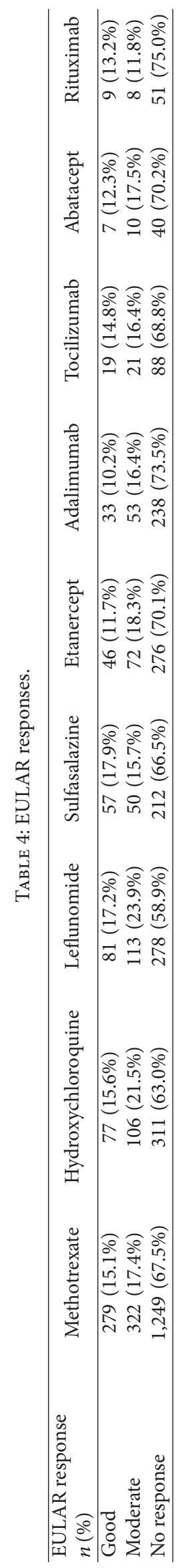


studies $[5,6,19-22]$. There are a number of reasons why this might be the case. For the RCTs the patient population was selected with a high disease activity, while the published observational studies had stricter selection criteria for the patients in their analyses. In our study the average bDMARD DAS28-ESR prior to medication changes was 3.4, which is considerably lower than the average DAS28-ESR seen in RCTs (often around 6.0) and observational registries (around 5.1-6.0) $[5,6,15-21]$ using the same medications. A higher starting DAS28 provides the opportunity for a larger fall in DAS28 following the commencement of a medication, and this may explain the differences between the OPAL and other reported bDMARD responses. We were unable to explain the observed lower baseline DAS28-ESR for patients starting bDMARDS; presumably the treating rheumatologist considered there was sufficient disease activity to warrant commencement of biologic treatment.

There are limitations in the ability to make comparisons between drugs in this study. Rather than being randomly allocated, medications were selected based on individual patient comorbidities and clinician perceptions of different medication efficacy/risk profiles. In addition, Australian government rules, which restrict funding support for bDMARDs until there is a documented failure to respond to at least 2 cDMARDs, may also play a role [2]. Because these and other variables are not able to be controlled in this study design, any outcome differences might be explained by differences in, for example, disease severity/refractoriness being unequally allocated in the treatment groups.

In conclusion, this longitudinal study in a large cohort of unselected community-based RA patients from OPAL clinics identified that medication choices in the Australian rheumatology clinics were in keeping with international guidelines. One exception was that there appeared to be less use of initial combination cDMARDs therapy. Treatment survival was long in all DMARDs groups studied. The first RA medication changes were escalations from monotherapy methotrexate, cDMARDs other than methotrexate, and bDMARDs. Improvements in disease activity were seen in all DMARDs studied. Initiation of cDMARDs/bDMARDs was associated with disease activity reduction 6 months after commencement of treatment.

\section{Ethical Approval}

This study was conducted with the approval of the University of New South Wales Human Research Ethics Committee.

\section{Conflicts of Interest}

In compliance with the Uniform Requirements for Manuscripts, established by the International Committee of Medical Journal Editors, the supporter of this study did not impose any impediment, directly or indirectly, on the publication of the study results. Lynden Roberts has been paid to provide expert advice to AstraZeneca, bioCSL, and Menarini and has received travel support from Roche to present the results of OPAL research. Kathleen Tymms reports nonfinancial support from Roche during the conduct of this study and consulting fees for advisory board activity outside the submitted work. Julien de Jager is a member of the Scientific Advisory Committee for Arthritis Australia, has served on advisory boards for Janssen, Celgene, participated in clinical trials supported by $\mathrm{UCB}$, and presented at meetings supported by Janssen and Amgen during the last 12 months. Geoffrey Littlejohn reports nonfinancial support from Roche during the conduct of the study and consulting fees for activities outside the submitted work from AbbVie, Bristol-Myers Squibb, Celgene, Pfizer, Roche, and UCB. Hedley Griffiths has received grant support from Roche, AbbVie, Janssen, and Bristol-Meyers Squibb and conference attendance sponsorship from Pfizer and served on advisory boards for Janssen, Bristol-Meyers Squibb, and Celgene, all outside the submitted work. Dave Nicholls did not report any conflicts of interest other than the nonfinancial support from Roche for this study. Paul Bird has no conflicts of interest to disclose. Jennifer Young is an employee of Roche Products Pty Limited. Julie Hill who is an employee of McCloud Consulting Group was contracted by Roche to support this research. Jane Zochling has received consulting fees for advisory board activity from Roche, Novartis, and Celgene for activities outside the submitted work and speaker fees from AbbVie.

\section{Acknowledgments}

This work was supported by Roche Products Pty Limited (Australia). The authors would like to acknowledge Dr. David Hoffman from S4S for assistance with data extraction. Medical writing was provided by Dr. Joseline Ojaimi from Roche. The authors would like to acknowledge contribution of data from OPAL members: Coast Joint Care; Barwon Rheumatology Service; Georgetown Arthritis; Combined Rheumatology Practice; Dr. Claire Barrett; Dr. Laurel Young; Rheumatology Tasmania; Dr. Anna Finniss; Hobart Specialists Group; Dr. Chris Mack; Hills Rheumatology; Dr. Daniel Lewis; Drs. Godfrey and Wong; Susan Street Specialists Centre; Dr. Mona Marabani; Dr. Chris Fong; Dr. Talib Tahir.

\section{References}

[1] C. Gaujoux-Viala, J. S. Smolen, R. Landewe et al., "Current evidence for the management of rheumatoid arthritis with synthetic disease-modifying antirheumatic drugs: a systematic literature review informing the EULAR recommendations for the management of rheumatoid arthritis," Annals of the Rheumatic Diseases, vol. 69, no. 6, pp. 1004-1009, 2010.

[2] S. L. Gorter, J. W. Bijlsma, M. Cutolo et al., "Current evidence for the management of rheumatoid arthritis with glucocorticoids: a systematic literature review informing the EULAR recommendations for the management of rheumatoid arthritis," Annals of the Rheumatic Diseases, vol. 69, no. 6, pp. 1010-1014, 2010.

[3] J. L. Nam, K. L. Winthrop, and R. F. van Vollenhoven, "Current evidence for the management of rheumatoid arthritis with biological disease-modifying antirheumatic drugs: a systematic literature review informing the EULAR recommendations for the management of RA," Annals of the Rheumatic Diseases, vol. 69, no. 6, pp. 976-986, 2010. 
[4] J. S. Smolen, R. Landewe, F. C. Breedveld et al., "EULAR recommendations for the management of rheumatoid arthritis with synthetic and biological disease-modifying antirheumatic drugs: 2013 update," Annals of the Rheumatic Diseases, vol. 73, no. 3, pp. 492-509, 2014.

[5] M. L. Hetland, I. J. Christensen, U. Tarp et al., "Direct comparison of treatment responses, remission rates, and drug adherence in patients with rheumatoid arthritis treated with adalimumab, etanercept, or infliximab: results from eight years of surveillance of clinical practice in the nationwide Danish DANBIO registry," Arthritis and Rheumatism, vol. 62, no. 1, pp. 22-32, 2010.

[6] F. Iannone, E. Gremese, F. Atzeni et al., "Longterm retention of tumor necrosis factor- $\alpha$ inhibitor therapy in a large Italian cohort of patients with rheumatoid arthritis from the GISEA Registry: an appraisal of predictors," Journal of Rheumatology, vol. 39, no. 6, pp. 1179-1184, 2012.

[7] G. Littlejohn, L. Roberts, M. Arnold et al., "A multi-center, observational study shows high proportion of Australian rheumatoid arthritis patients have inadequate disease control," International Journal of Rheumatic Diseases, vol. 16, no. 5, pp. 532-538, 2013.

[8] K. Tymms, J. Zochling, J. Scott et al., "Barriers to optimal disease control for rheumatoid arthritis patients with moderate and high disease activity," Arthritis Care and Research, vol. 66, no. 2, pp. 190-196, 2014.

[9] A. M. van Gestel, M. L. Prevoo, M. A. van't Hof, M. H. van Rijswijk, L. B. van de Putte, and P. L. van Riel, "Development and validation of the European League against rheumatism response criteria for rheumatoid arthritis: comparison with the preliminary American College of Rheumatology and the World Health Organization/International League Against Rheumatism Criteria," Arthritis and Rheumatism, vol. 39, no. 1, pp. 3440, 1996.

[10] A. Finckh, S. Dehler, and C. Gabay, "The effectiveness of leflunomide as a co-therapy of tumour necrosis factor inhibitors in rheumatoid arthritis: a population-based study," Annals of the Rheumatic Diseases, vol. 68, no. 1, pp. 33-39, 2009.

[11] S. C. Kim, E. Yelin, C. Tonner, and D. H. Solomon, "Changes in use of disease-modifying antirheumatic drugs for rheumatoid arthritis in the United States during 1983-2009," Arthritis Care and Research, vol. 65, no. 9, pp. 1529-1533, 2013.

[12] E. G. Favalli, F. Pregnolato, M. Biggioggero et al., "Twelveyear retention rate of first-line tumor necrosis factor inhibitors in rheumatoid arthritis: real-life data from a local registry," Arthritis Care and Research, vol. 68, no. 4, pp. 432-439, 2016.

[13] J. A. Singh, D. E. Furst, A. Bharat et al., "2012 update of the 2008 American College of Rheumatology recommendations for the use of disease-modifying antirheumatic drugs and biologic agents in the treatment of rheumatoid arthritis," Arthritis Care and Research, vol. 64, no. 5, pp. 625-639, 2012.

[14] NICE, "Rheumatoid arthritis. The management of rheumatoid arthritis in adults. NICE clinical guideline 79," 2013, https:// www.nice.org.uk/guidance/cg79.

[15] C. Gabay, P. Emery, R. van Vollenhoven et al., "Tocilizumab monotherapy versus adalimumab monotherapy for treatment of rheumatoid arthritis (ADACTA): a randomised, doubleblind, controlled phase 4 trial," Lancet, vol. 381, no. 9877, pp. 1541-1550, 2013.

[16] H. Kameda, Y. Ueki, K. Saito et al., "Etanercept (ETN) with methotrexate (MTX) is better than ETN monotherapy in patients with active rheumatoid arthritis despite MTX therapy: a randomized trial," Modern Rheumatology, vol. 20, no. 6, pp. 531-538, 2010.

[17] M. Schiff, M. Keiserman, C. Codding et al., "Efficacy and safety of abatacept or infliximab versus placebo in ATTEST: a phase III, multi-centre, randomised, double-blind, placebocontrolled study in patients with rheumatoid arthritis and an inadequate response to methotrexate," Annals of the Rheumatic Diseases, vol. 67, no. 8, pp. 1096-1103, 2008.

[18] L. B. van de Putte, C. Atkins, M. Malaise et al., "Efficacy and safety of adalimumab as monotherapy in patients with rheumatoid arthritis for whom previous disease modifying antirheumatic drug treatment has failed," Annals of the Rheumatic Diseases, vol. 63, no. 5, pp. 508-516, 2004.

[19] H. Forsblad-d'Elia, K. Bengtsson, L. E. Kristensen, and L. T. Jacobsson, "Drug adherence, response and predictors thereof for tocilizumab in patients with rheumatoid arthritis: results from the Swedish biologics register," Rheumatology, vol. 54, no. 7, pp. 1186-1193, 2015.

[20] H. C. Leffers, M. Ostergaard, B. Glintborg et al., "Efficacy of abatacept and tocilizumab in patients with rheumatoid arthritis treated in clinical practice: results from the nationwide Danish DANBIO registry," Annals of the Rheumatic Diseases, vol. 70, no. 7, pp. 1216-1222, 2011.

[21] C. Pease, J. E. Pope, D. Truong et al., "Comparison of anti-tnf treatment initiation in rheumatoid arthritis databases demonstrates wide country variability in patient parameters at initiation of anti-tnf therapy," Seminars in Arthritis and Rheumatism, vol. 41, no. 1, pp. 81-89, 2011.

[22] T. S. Jorgensen, L. E. Kristensen, and R. Christensen, "Effectiveness and drug adherence of biologic monotherapy in routine care of patients with rheumatoid arthritis: a cohort study of patients registered in the Danish biologics registry," Rheumatology, vol. 54, no. 12, pp. 2156-2165, 2015. 


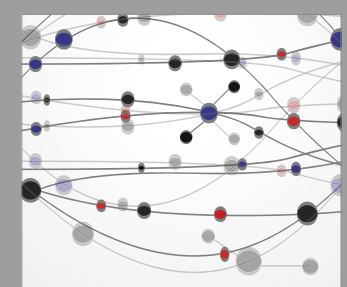

The Scientific World Journal
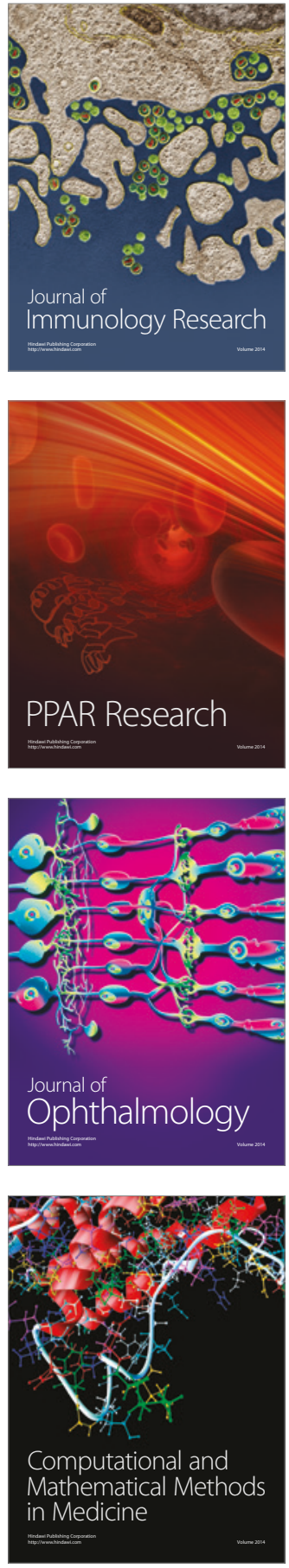

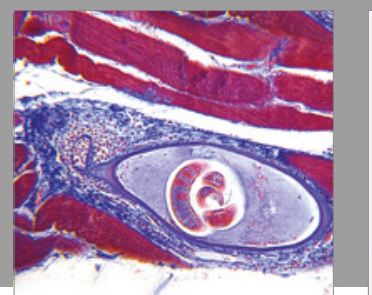

Gastroenterology Research and Practice
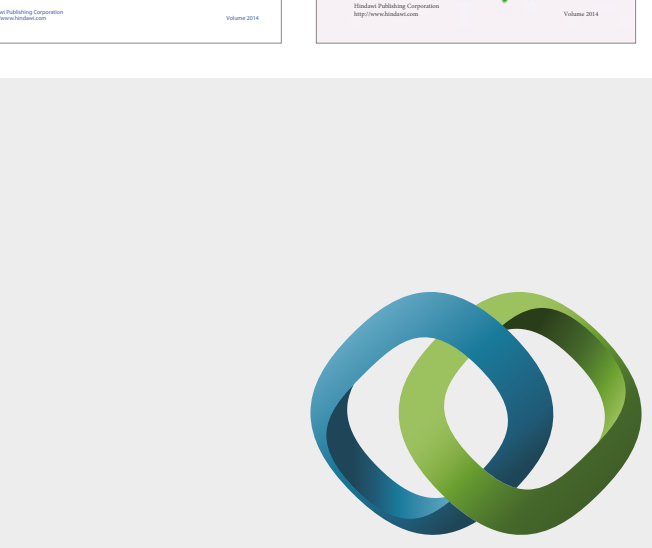

\section{Hindawi}

Submit your manuscripts at

https://www.hindawi.com
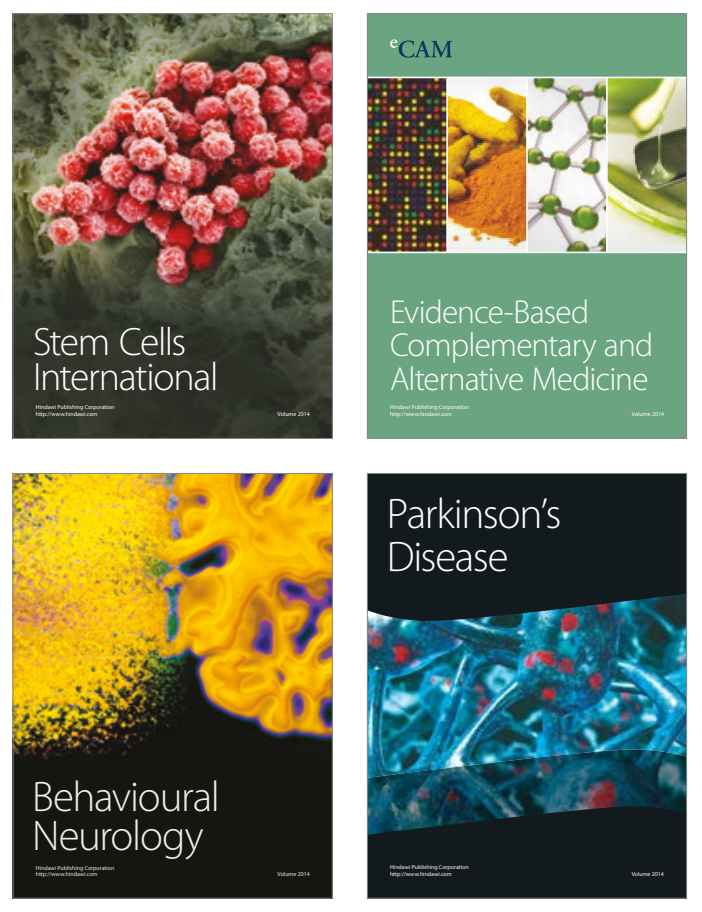
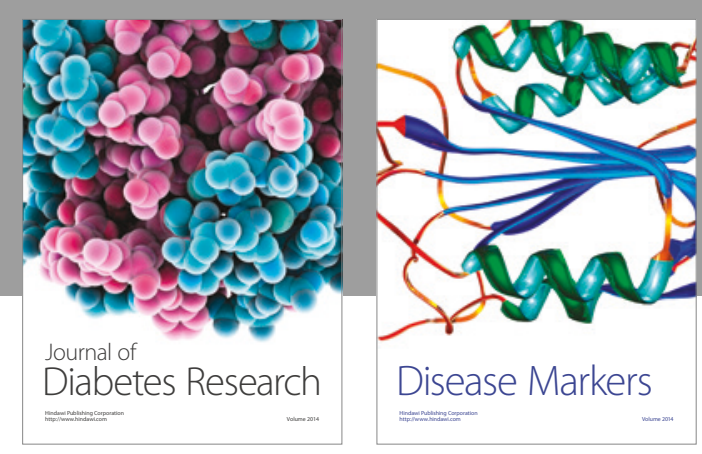

Disease Markers
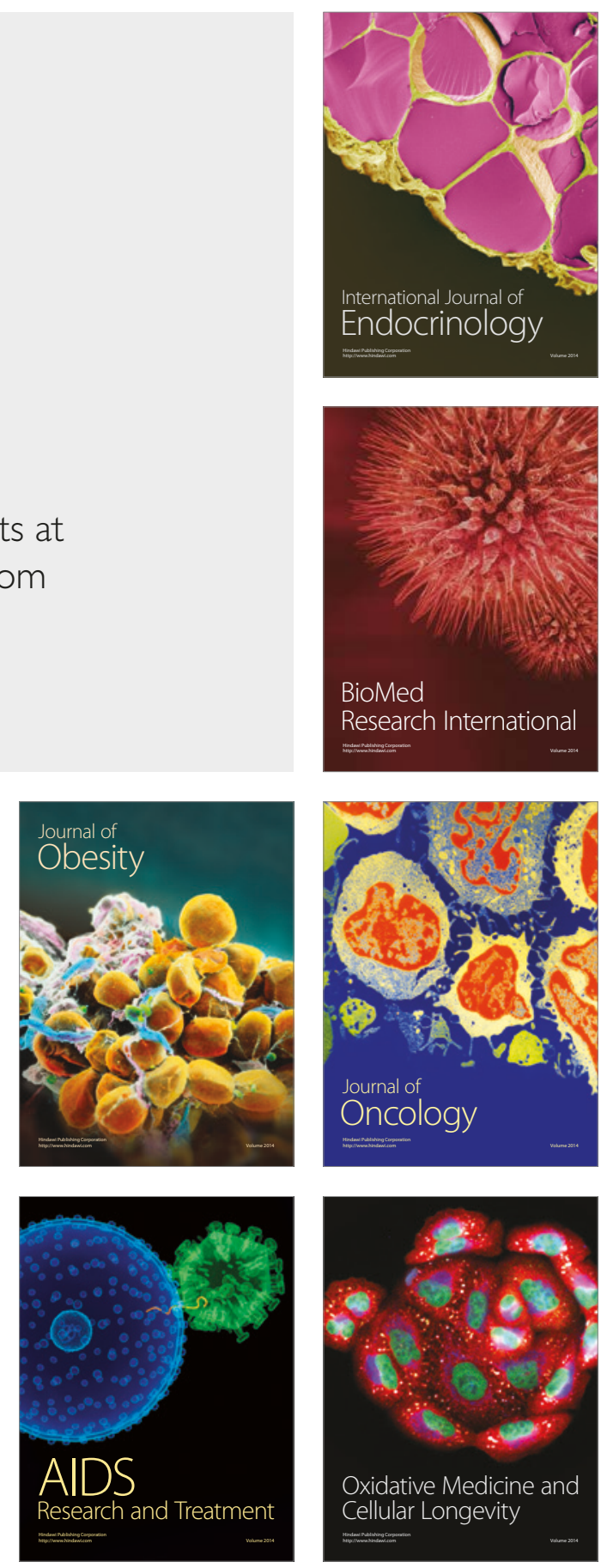